\title{
ESPECIES NUEVAS Y NUEVOS REGISTROS DE ABEJAS PARA LAS ANTILLAS \\ (HYMENOPTERA: ANTHOPHILA; COLLETIDAE, HALICTIDAE)
}

\begin{abstract}
Julio A. Genaro
Investigador Asociado del Museo Nacional de Historia Natural "Prof. Eugenio de Jesús Marcano". Calle César Nicolás Penson, Plaza de la Cultura, Juan Pablo Duarte Santo Domingo, 10204, República Dominicana.polimita@hotmail.com
\end{abstract}

\section{RESUMEN}

La región antillana se considera un punto caliente de la biodiversidad, debido al alto nivel de endemismo de la biota y a la cada vez más creciente pérdida del hábitat. Muchas especies todavía permanecen sin ser descubiertas, mientras que otras depositadas en las colecciones de insectos esperan por su estudio y descripción. Cuatro especies nuevas de abejas antillanas se describen e ilustran: Colletidae, Hylaeus dearmasi sp. nov. (Cuba); H. hispaniolensis sp. nov. (Hispaniola); Halictidae, Lasioglossum obamai sp. nov. (Cuba); Augochlora boriquena sp. nov. (Puerto Rico). Una lista anotada de las especies antillanas de Hylaeus, incluyendo nombres sinónimos y distribución es presentada. Hylaeus (Hylaeana) royesi Raw y H. (Hylaeana) formosus Krombein se registran por primera vez para Cuba.

Palabras clave: taxonomía, registros nuevos, Hylaeus, Lasioglossum, Augochlora, Cuba, Hispaniola, Puerto Rico.

Title: New species and new records of bees from the Antilles (Hymenoptera: Anthophila; Colletidae, Halictidae).

\section{ABSTRACT}

The Antillean region is considered a threatened biodiversity hotspot because of its high levels of endemism and growing habitat destruction. Still most species remain to be discovered while others await description in entomological collections. Four new species of Antillean bees are described and illustrated: Colletidae, Hylaeus dearmasi sp. nov. (Cuba); H. hispaniolensis sp. nov. (Hispaniola); Halictidae, Lasioglossum obamai sp. nov. (Cuba); Augochlora boriquena sp. nov. (Puerto Rico). A checklist of the species in the genus Hylaeus from the Antilles is presented, including synonyms and distributional summaries. Hylaeus (Hylaeana) royesi Raw and H. (Hylaeana) formosus Krombein are reported for the first time from Cuba.

Keywords: taxonomy, new records, Hylaeus, Lasioglossum, Augochlora, Cuba, Hispaniola, Puerto Rico.

\section{INTRODUCCIÓN}

El tema de los polinizadores nativos (en el cual las abejas son un componente de peso) ha adquirido vigencia debido a los efectos del cambio climático en el planeta, causado por las transformaciones del hombre y el crecimiento acelerado de su población. Muchas especies están desapareciendo sin que lleguemos a conocerlas.

El género Hylaeus está constituido por abejas de tamaño pequeño y escasamente recolectadas. Muchas especies antillanas se conocen por uno o pocos especímenes, o por un solo sexo. 
El tamaño pequeño y el hábito de nidificar en el interior de ramitas secas, favorece la dispersión fundamentalmente por la acción de los vientos. Por esta razón, una especie puede encontrarse en diferentes islas antillanas, aunque hemos observado endemismo en algunas formas. Urban y Moure (2007), en el catálogo de las abejas Neotropicales, presentan el listado más reciente de las especies de Hylaeus.

\section{OBJETIVOS}

-Describir cuatro especies nuevas de abejas de Cuba, la Hispaniola y Puerto Rico, para que se conozca su presencia, con vistas a la conservación.

-Presentar una lista anotada de las especies antillanas de Hylaeus incluyendo registros nuevos.

\section{MATERIALES Y MÉTODOS}

La terminología utilizada para la escultura de la superficie del tegumento sigue a Harris (1979). Las abreviaturas son usadas como sigue: cada tergo o esterno metasomal es llamado $\mathrm{T}$ o S, respectivamente, seguido por un número que lo define específicamente, ejemplo: T1 es el primer tergo y $\mathrm{S} 1$ el primer esterno. La densidad de las puntuaciones está dada en términos de la relación entre el diámetro de la puntuación (d) y el inter-espacio (i) entre ellas, como ejemplo $\mathrm{i}=2 \mathrm{~d}$, para demostrar su separación. La longitud de los pelos está en relación con el diámetro del ocelo medio (DOM), por ejemplo $<$ DOM significa que la longitud del pelo es menor que el DOM.

Instituciones donde está depositado el material estudiado o a las cuales se hace referencia en el texto: CNC, Canadian National Collection of Insects, Arachnids and Nematodes, Ottawa; JAG, colección personal del autor; LACM, Natural History Museum of Los Angeles County, Estados Unidos; NMNH, National Museum of Natural History, Estados Unidos, Institución Smithsoniana y UPRM, Universidad de Puerto Rico, recinto Mayagüez.

\section{TAXONOMÍA}

Orden Hymenoptera, Superfamilia Apoidea,

Familia Colletidae, Subfamilia Hylaeinae.

Hylaeus (Prosopis) dearmasi sp. nov.

(Figuras 1-5)

Diagnosis. Tamaño pequeño, con cara extensivamente manchada de blanco (Fig. 1); área basal del propodeo granulado, con estrías cortas casi paralelas en el margen anterior (Fig. 2) (en un ejemplar están poco definidas) y T1 con puntuaciones definidas, mayores que en el resto de los tergos.

Una combinación de caracteres como el patrón de marcas blancas, la esculturación del propodeo, puntuación de T1, la forma de S7 y S8 diferencia a ésta de las especies antillanas conocidas. Hylaeus limbifrons (Cresson, 1869), cuya terminalia es desconocida, es una especie de mayor tamaño, con esculturación grosera en el propodeo y T1 pulido, con pocas puntuaciones, pequeñas.

Diagnosis. Small size, with face largely mark of white (Fig. 1); basal area of propodeum granulate, with short, subparallel striae at anterior margin (Fig. 2) (poor defined in one specimen); T1 with defined punctures, larger than in the rest of terga. 
A combination of characters such as pattern of white marks, surface sculpturing of propodeum; puncturation of T1, and shape of S7 and S8 separates the new species from the rest of the Antillean species. Hylaeus limbifrons (Cresson, 1869) which terminalia is unknown is a larger species, with coarse sculpturation on propodeum, and T1 polished, with scattered small punctuations.

\section{DESCRIPCIÓN}

Macho. Longitud corporal total: 4.0-4.3 mm. Longitud ala anterior: $3.0-3.5 \mathrm{~mm}$.

Coloración. Tegumento negro en cabeza y mesosoma, excepto blanco hueso en las siguientes áreas: parte de las mandíbulas, labro, clípeo, área paraocular (Fig. 1), mancha en lóbulo pronotal (solo en algunos ejemplares), mancha que ocupa la mitad anterior de la tégula (presente en algunos ejemplares), banda en pronoto observada solamente en dos ejemplares: interrumpida en el medio en un ejemplar y entera en el otro; borde ventral del área supra-clipeal (llega a ser castaño-rojizo en algunos ejemplares).

Antenas castaño oscuro, ligeramente más claras ventralmente. Alas hialinas con nervaduras castaño. Patas castaño oscuro, con áreas castaño amarillo claro en tarsos, sobre todo en protibia, un tercio del área dorsal en mesotibia y mitad dorsal de metatibia. Metasoma castaño, siendo más rojizo ventralmente.

Esculturación. Cabeza. Clípeo, área supraclipeal y área paraocular con puntuaciones aisladas, variables ( $\mathrm{i}=1-3.5 \mathrm{~d})$; área supra-antenal puntuada-reticulada, esparciéndose hacia el vértex ( $\mathrm{i}=0-1.5 \mathrm{~d})$ y reticulándose hacia el margen posterior del vértex, impuntuada sobre los alveólos antenales; gena costulada, con puntuaciones unidas, alargadas, formando hileras. Línea frontal que llega hasta la mitad del área supraclipeal, que es elevada.

Mesosoma. Mesoescuto con puntuaciones profundas, variables ( $\mathrm{i}<\mathrm{d}$; $\mathrm{i}=0.5-2 \mathrm{~d})$; escutelo con puntuaciones mayores, variables, más esparcidas $(\mathrm{i}=1-3 \mathrm{~d})$; metanoto granulado, con pocas puntuaciones, variables, muy esparcidas. Pre-episterno y mesepisterno con puntuaciones variables $(\mathrm{i}=0.5-2.5 \mathrm{~d})$, similar a la gena en su tercio superior; metepisterno costado en la mitad superior y puntuaciones profundas, unidas en la mitad inferior. Superficie posterior del propodeo punteada-reticulada; triángulo del propodeo granulado, con estrías cortas, casi paralelas sobre el margen anterior (Fig. 2) (casi no observables en el ejemplar de Bahía de Guantánamo).

Metasoma. Tergos con puntuaciones de menor tamaño que el resto del cuerpo, mayores en T1 (i=1-4d), disminuyendo el tamaño en T2 y algo menores en el resto de los tergos; esternos granulados, con puntuaciones mayores que en los tergos, aisladas, variables, con una seta que emerge de cada puntuación.

Pubescencia. Blanca. Pelos simples, cortos $(<\mathrm{DOM})$, algo esparcidos en cabeza y mesosoma; ligeramente más largos $(=\mathrm{DOM})$ en S4-S6. Pelos plumosos cortos $(<\mathrm{DOM})$ que bordean el mesoescuto (visible en ejemplares mejor preservados). Parche apicolateral en T1 de pelos ramificados, recostados, cortos $(<\mathrm{DOM})$ (observable en ejemplares mejor conservados); parche apicolateral en T2 menos denso, con pelos simples; pelos ramificados, largos (=1.5 DOM), aislados sobre metanoto y pre-episterno; más cortos sobre superficie posterior del propodeo $(<\mathrm{DOM})$; pelos largos (=1.5 DOM), ramificados, aislados en área apicolateral de últimos tergos. 
Estructura. Tercer segmento antenal más ancho que largo, menor que el cuarto (casi la mitad de su longitud).

Terminalia. Genitales: pelos plumosos, curvos, largos (=2DOM) en el ápice de los gonostilos; valvula del pene con ápice estilizado y curvado (Fig. 3). S7: lóbulos apicales pequeños con los brazos apodemales extendiéndose anteriormente (Fig. 4). S8: proceso apical algo alargado, ensanchado y cóncavo en el ápice; apodemas laterales con apodema angulado sobre el margen antero-lateral (Fig. 5).

Tipos. Holotipo. CUBA, Archipiélago Jardines de La Reina, cayo Caballones, iv.1971, col. L. F. de Armas (1 macho, NMNH). Paratipos: CUBA, Archipiélago Jardines de La Reina, cayo Anclitas, iv.1971, col. L. F. de Armas (5 machos, JAG, CNC); cayo Caballones, iv.1971, col. L. F. de Armas (2 machos, JAG); Bahía de Guantánamo, 19.9168N 75.1033W, 6-13.vii.2010, col. J. Montalvo, USGS-DRO, 179499 (1 macho, NMNH).

Etimología. Dedicada al amigo y eminente zoólogo cubano Luis F. de Armas, recolector de gran parte de la serie tipo.

Comentario. Luis F. de Armas (com. pers., 3.i.2016) ofreció detalles sobre las circunstancias de la recolecta de la especie nueva: "Cayo Caballones está a $2.3 \mathrm{~km}$ al oeste de cayo Anclita, separado por un estrecho canal. En Anclita recolecté en el extremo occidental, mientras que en Caballones lo hice durante unas pocas horas en el extremo oriental; exclusivamente pase la red entomológica por la vegetación herbácea de la costa y sus alrededores, donde se observa una vegetación xerofítica". Otros datos sobre las características del hábitat aparecen en Armas (1976).

Hembra. Desconocida.

Distribución. Cuba y posiblemente Las Bahamas (Isla San Salvador). Según R. R. Snelling (com. pers., 1999) un macho recolectado en Las Bahamas pudiera ser conespecífico.

\section{Hylaeus (Prosopis) hispaniolensis sp. nov.}

(Figuras 6-8)

Diagnosis. Especie grande, robusta, con tegumento negro y algunas manchas blancas en el cuerpo; dos franjas blancas y estrechas en área paraocular, que no llegan al alvéolo antenal (Fig. 6). Área basal del propodeo con estriaciones subparalelas, reticulado a los lados; área posterior del propodeo granulosa con puntuaciones; triángulo formado en área central granuloso (Figs. 7, 8). T1 pulido, con el disco impuntuado.

De las especies antillanas, la otra especie robusta con la que pudiera confundirse es H. limbifrons, de Cuba, de la cual se separa por la extensión de las franjas blancas de la cara, ya que en la especie cubana son más anchas, llegando a tocar el alvéolo antenal. Hylaeus limbifrons presenta el área posterior del propodeo con esculturación más grosera, mientras que en la especie nueva es granulosa.

Diagnosis. Large size, robust, black with white marks; two narrow white stripes on paraocular area that does not reach the antennal sockets (Fig. 6). Basal area of propodeum with subparalell striae, reticulate on sides, granulate at the middle; posterior surface of propodeum granulate with punctures (Figs. 7, 8). T1 polished, with disc impunctate. 
Among the Antillean species, $H$. limbifrons, from Cuba, is another robust bee that can be mistaken with the new species. The new species can be identified by its narrow stripes on the face as opposed to $H$. limbifrons' wider stripes that does reach the antennal socket. In addition, the Cuban species has the basal area of propodeum with coarser sculpturation.

\section{DESCRIPCIÓN}

Hembra. Longitud corporal total: $7.3 \mathrm{~mm}$. Longitud ala anterior: $4.8 \mathrm{~mm}$.

Coloración. Tegumento negro, con las siguientes partes blanco hueso: área paraocular; pronoto (banda interrumpida en el centro); lóbulo pronotal; mitad anterior tégula; extremo basal externo de las tibias (alargándose más en la metatibia). Alas ahumadas (castaño claro) con venación negra.

Esculturación. Cabeza. Clípeo, área supraclipeal y área paraocular inferior granulosas con puntuaciones grandes, variables $(\mathrm{i}=0-3.5 \mathrm{~d})$; área paraocular superior con puntuaciones alargadas, unidas; frente punteada-reticulada; vértex coráceo con puntuaciones variables $(i=0-1 d)$; gena corácea con puntuaciones variables $(i=0.5-2 d)$. Mesosoma. Mesoescuto granulado con puntuaciones variables (más esparcidas en el disco, $\mathrm{i}=0.5-5 \mathrm{~d}$ ); escutelo y metanoto granuloso con puntuaciones variables $(\mathrm{i}=0.5-3 \mathrm{~d})$. Área basal del propodeo con estriaciones subparalelas, reticulado a los lados; área posterior del propodeo granulosa con puntuaciones variables $(\mathrm{i}=0.5-3 \mathrm{~d})$, triángulo formado en área central granuloso (Fig. 8). Pre-episterno y mesespisterno granuloso con puntuaciones variables $(i=0.5-1.5 \mathrm{~d})$; metepisterno con estrías paralelas, puntuaciones variables. Metasoma con puntuaciones finas, mucho menores que el resto del cuerpo. T1 pulido, brillante, con disco impuntuado; puntuaciones variables $(i=1-4 d)$ hacia los lados y margen posterior. T2-T6 con puntuaciones variables $(i=0-4.5 d)$. Esternos con puntuaciones variables $(i=0.5-4.5 \mathrm{~d})$, ligeramente más profundas.

Pubescencia. Blanca excepto pelos negros al final del metasoma. Pelos cortos variables $(\leq \mathrm{DOM})$, aislados en el clípeo, que emergen de cada puntuación; pelos algo más largos, ramificados en el área supra-antenal. Pelos cortos $(<\mathrm{DOM})$, simples en mesoescuto y escutelo, ligeramente más largos en áreas del episterno. Pelos más largos ( $>$ DOM) y ramificados en metanoto y superficie posterior del propodeo. Patas con pubescencia corta $(<\mathrm{DOM})$ simple. Banda apical en T1-T4 de pelos densos, ramificados, interrumpida en el centro. Esternos con pelos cortos $(=1 / 2 \mathrm{DOM})$ aislados. Pelos largos (>DOM), negros, ramificados en ápice del metasoma.

Estructura. Margen del clípeo ligeramente cóncavo. Tercer segmento antenal tan largo como ancho (algo más largo que el cuarto).

Tipos. Holotipo hembra. La Hispaniola, República Dominicana, Parque Nacional A. Bermúdez, Antón Sape Bueno, Trillo de La Hispaniola, xii.2008, col. J. A. Genaro (1 hembra, NMNH).

Etimología. Nombrada por la isla donde se recolectó la especie, La Hispaniola.

Comentario. Constituye la especie vicariante de $H$. limbifrons. Otros ejemplos de especies vicariantes entre Cuba y La Hispaniola son mencionados por Genaro (2009).

Macho. Desconocido.

Distribución. La Hispaniola. 


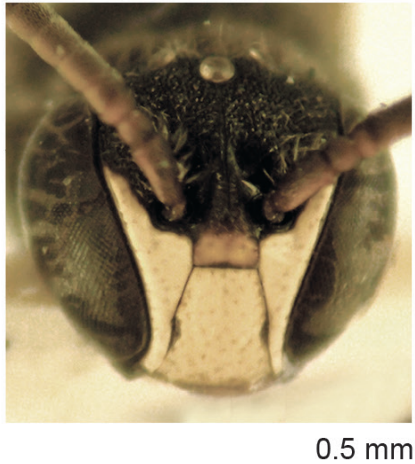

1
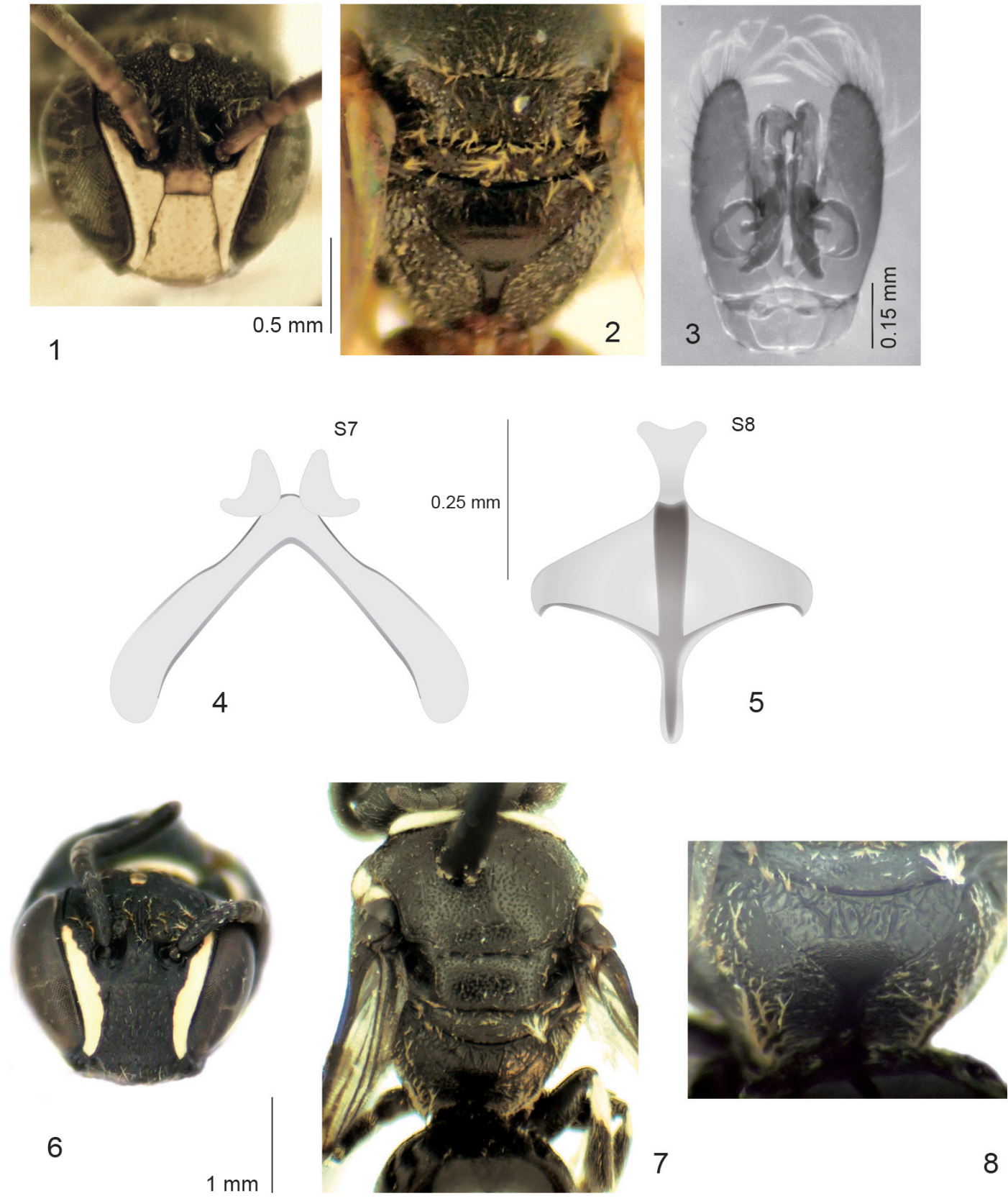

7

Figuras 1-8. Especies antillanas nuevas del género Hylaeus. 1-5, Hylaeus dearmasi macho: 1, vista frontal de la cabeza; 2, vista parcial del mesosoma mostrando el propodeo; 3, cápsula genital en vista dorsal; 4, S7; 5, S8. 6-8. Hylaeus hispaniolensis hembra: 6, vista frontal de la cabeza; 7 , mesosoma en vista dorsal; 8, propodeo. 


\section{Familia Halictidae, Subfamilia Halictinae, Tribu Halictini Lasioglossum (Dialictus) obamai sp. nov.}

(Figuras 9-12, 15, 16)

Lasioglossum sp. E. Alayo, 1973. Catálogo de los himenópteros de Cuba, p. 200. Macho.

Lasioglossum sp. E. Alayo, 1976. Serie Biológica, Cuba 68: 20. Macho.

Diagnosis. Tegumento verde-azul metálico. Gena angulada, con margen triangular; área basal del propodeo granulosa, con estrías subparalelas que no llegan al margen posterior. S8 carente de proceso medio; lóbulo membranoso retroverso presente en gonostilo, pero reducido, distalmente alargado, fino. Una combinación de caracteres que incluyen la forma de la gena, esculturación del propodeo y de forma general la esculturación del mesosoma, separan esta especie de las más similares: L. havanense (Baker, 1906), L. engeli Genaro, 2001 (Fig. 13) y L. sierramaestrense Genaro, 2001 (Fig. 14).

Diagnosis. Green-blue metallic. Angulate genae, with triangular margin; basal area of propodeum granulate, with subparalelled striae not attaining posterior margin. S8 without median process; retrorse lobe, on ventral surface of genitalia, reduced, enlarged distally. A combination of characters such as gena's angle, sculpturation of mesosoma (mostly the sculpturation of propodeum) separates the new species from the similar ones: L. havanense (Baker, 1906), L. engeli Genaro, 2001 (Fig. 13) and L. sierramaestrense Genaro, 2001 (Fig. 14).

\section{DESCRIPCIÓN}

Macho. Longitud corporal total: 5.6-5.8 mm. Longitud ala anterior: 4.2-4.3 mm.

Coloración. Tegumento del cuerpo verde-azul metálico (Fig. 9). Antena castaño oscuro; patas y esternos castaño-rojizo con reflejos verde-azul metálico; venación del ala castaño, estigma castaño-rojizo.

Esculturación. Cabeza. Tegumento granuloso-puntuado con puntuaciones variables (i=0.5-5d) en la cara; más separadas y menos profundas en la frente; gena costulada con puntuaciones dispersas. Mesosoma. Puntuaciones variables en mesoescuto, dispersas en el disco ( $\mathrm{i}=0.5-2 \mathrm{~d})$; más esparcidas en escutelo $(i=0.5-4 d)$; metanoto con puntuaciones variables $(i=0-4 d)$ y estrías subparalelas. Área basal del propodeo granulosa, con estrías subparalelas que no llegan al margen posterior (Fig. 11); el número de estrías pueden variar con el individuo (el holotipo presenta pocas). Superficie posterior del propodeo granulosa, con puntuaciones escasas, dispersas; lateralmente es costulada-granulosa con puntuaciones variables $(\mathrm{i}=\mathrm{d} ; \mathrm{i}=0.5-4 \mathrm{~d})$. Pre-episterno rugoso, con algunas puntuaciones. Mesepisterno granuloso con puntuaciones muy grandes, variables $(\mathrm{i}=1-4 \mathrm{~d})$, poco profundas. Área hipoepimeral con puntuaciones más profundas, dispersas. Área dorsal del metepisterno con estrías paralelas, granulado con puntuaciones dispersas, ventralmente. Metasoma con puntuaciones menores. T1 anteriormente glabro, pulido, con puntuaciones aisladas, variables en área posterior $(\mathrm{i}=1-7 \mathrm{~d})$; el resto de los tergos con puntuaciones variables $(\mathrm{i}=0.5-4 \mathrm{~d})$. Esternos coriáceos, con puntuaciones aisladas.

Pubescencia. Blanca. Pelos aislados, ramificados en cabeza y mesosoma (=1-1.5 DOM), más largos sobre las mandíbulas (=1.8 DOM). Pelos simples, cortos $(<\mathrm{DOM})$ en área del disco de los tergos; más largos (>1.5-2.5 DOM), plumosos hacia los lados. 


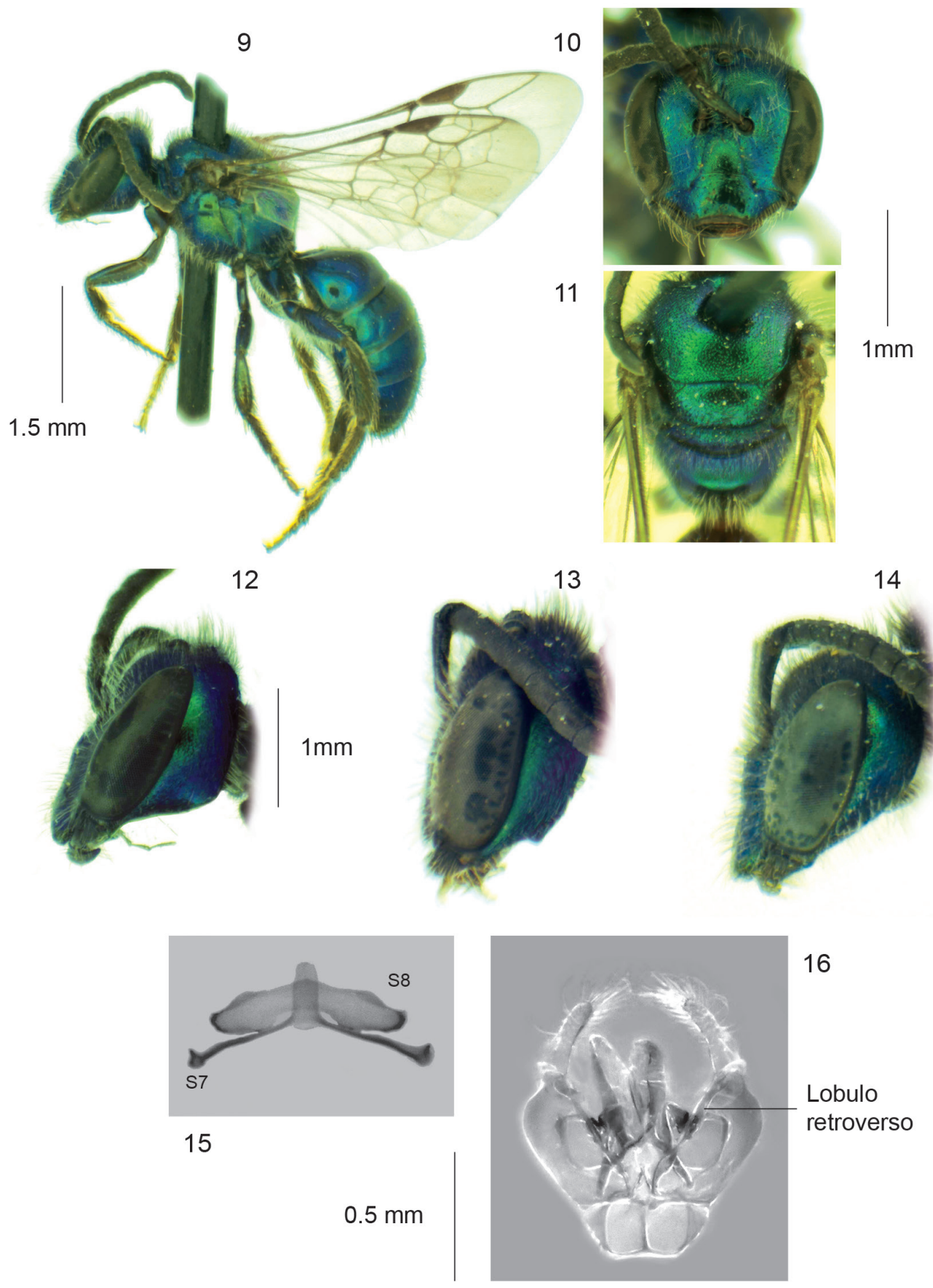

Figuras 9-16. Especies de Lasioglossum. 9-12, Lasioglossum obamai sp. nov. 9, habitus; 10, vista frontal de la cabeza; 11, vista dorsal del mesosoma. 12-14, Vista lateral de la cabeza. 12, L. obamai sp. nov; 13, L. engeli; 14, L. sierramaestrense. 15-16, Terminalia de L. obamai sp. nov. 15, esternos S7 (debajo) y S8 (arriba); 16, cápsula genital en vista ventral. 
Estructura. Margen del clípeo ligeramente cóncavo; ojo en su parte media ligeramente más ancho que la gena; gena angulada, con margen triangular (Fig. 12). Pedicelo y primer flagelómero antenal casi de la misma longitud (pedicelo ligeramente más largo). Angulo dorsolateral del pronoto recto, proyectándose bruscamente.

Terminalia. S7 carente de proceso medio alargado; S8 con proceso medio alargado, como en muchas especies de este género (Fig. 15). Genitalia. Gonostilo romo con setas alargadas en el extremo; lóbulo membranoso retroverso en la superficie ventral del gonostilo, presente, proyectándose ventralmente y expandiéndose posteriormente (Fig. 16).

Tipos. Holotipo macho. CUBA, La Gran Piedra, Oriente [Santiago de Cuba], vii. 1956, col. P. Alayo (NMNH). Paratipos machos. La Gran Piedra, Oriente [Santiago de Cuba], vii. 1956, col. P. Alayo, Lasioglossum sp. E (NMNH, JAG); La Gran Piedra, Oriente, vii.1963, cols. F. de Zayas, P. Alayo e I. García, ng, ns Dialictus sp. E, det. G. C. Eickwort (JAG); Loma del Gato, Oriente, vii.1953, col. F. de Zayas y P. Alayo, Habralictellus n. sp. 4, det. M. Engel (JAG).

Etimología. Especie dedicada al cuadragésimo cuarto presidente de los Estados Unidos de Norteamérica, Barack Obama, por su contribución a la conservación del medio ambiente (y los polinizadores nativos) y el mejoramiento en las relaciones con Cuba, entre otras políticas y reformas progresistas llevadas a cabo por su administración.

Hembra. Desconocida.

Distribución. Conocida hasta el momento de la Sierra Maestra, sudeste de Cuba.

\author{
Familia Halictidae, Subfamilia Halictinae, Tribu Augochlorini \\ Augochlora (Augochlora) boriquena sp. nov.
}

(Figuras 17-19)

Diagnosis. Tamaño pequeño. Cabeza, mesosoma y metasoma verde metálico, con reflejos dorados, sin tintes de azul. Augochlora buscki Cockerell, 1910 es la única especie del género conocida para Puerto Rico, la cual es de mayor tamaño, ambos sexos tienen un pequeño tubérculo en la gena que varía su desarrollo de acuerdo al individuo y está ausente en la especie nueva. Una combinación de caracteres como la forma de la gena, esculturación del propodeo, tamaño y color del tegumento la separan de otras especies antillanas. El color verde metálico de los tergos, con reflejos metálicos, la separa del resto de las especies.

Diagnosis. Small size. Head, mesosoma and metasoma metallic green with gold reflections without bluish tint. Augochlora buscki Cockerell, 1910 is the only species of the genus known from Puerto Rico, which is bigger. Both sexes of $A$. buscki have a small variable tubercle on gena (absent in the new species). A combination of characters such as size, shape of the gena, sculpturation of propodeum, and its mostly bright green color on the metasoma separates $A$. boriquena sp. nov. from the rest of the antillean species.

\title{
DESCRIPCIÓN
}

Hembra. Longitud corporal total: 6.6-7.8 mm. Longitud ala anterior: 4.9-5.2 $\mathrm{mm}$.

Coloración. Tegumento del cuerpo verde metálico con reflejos dorados, excepto: castaño oscuro en las mandíbulas, margen del clípeo, labro, escapo, pedicelo, primer segmento antenal (el resto castaño claro), patas (con ligero reflejo verde metálico) y esternos abdominales 


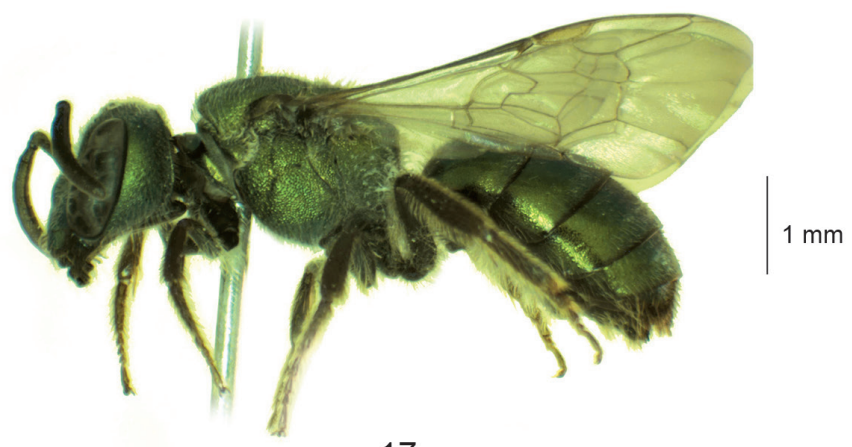

17
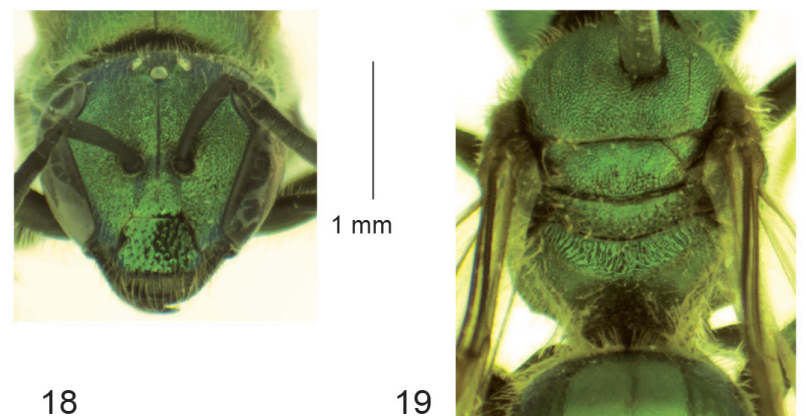

18

19

Figuras 17-19. Augochlora boriquena sp. nov. Hembra. 17, habitus; 18, vista frontal de la cabeza; 19, vista dorsal del propodeo.

(con ligeros reflejos metálicos); castaño claro en tarsos, nervaduras del ala y estigma (excepto C y Sc+R que son castaño oscuro).

Esculturación. Cabeza. Clípeo con puntuaciones irregulares (i=0-1.5d), tendiendo a alargarse, más unidas a los lados, mayores que las del resto de la cabeza. Area supraclipeal con puntuaciones irregulares $(\mathrm{i}=0-2 \mathrm{~d})$; área paraoculary frente puntuado-arrugado volviéndose puntuado-reticulado hacia el vértex y mitad anterior de la gena; la otra mitad de la gena granulosa; vértex arrugado. Mesosoma. Mesoescuto irregularmente puntuado ( $\mathrm{i}=0-1.5 \mathrm{~d}$ ); escutelo con puntuaciones grandes y pequeñas mostrando áreas arrugadas-lacunosas. Metanoto arrugado con puntuaciones aisladas; área basal del propodeo con surcos subparalelos (ramificadas en ocasiones) que llegan a tocar el margen posterior (Fig. 19); los surcos centrales se ramifican transversalmente (variable entre individuos). Superficie posterior del propodeo arrugada con puntuaciones grandes aisladas; ligeramente estriada en el área ventral, centralmente. Pre-episterno y mesepisterno puntuado-reticulado; metepisterno con puntuaciones unidas, estriado horizontalmente en área dorsal. Metasoma. Tergos con puntuaciones pequeñas irregulares ( $\mathrm{i}=1-4 \mathrm{~d})$. Esternos granulados con puntuaciones ligeramente mayores que en los tergos, irregularmente puntuados.

Pubescencia. Pubescencia corporal compuesta de pelos ramificados, blanca, excepto en las patas donde es blanco-amarillo. Pelos (=DOM ó =1.2 DOM) en vértex, más largos sobre el margen del clípeo y base mandíbulas (=1.5 DOM); corta en mesosoma (=DOM), algo más larga en escutelo. Ligeramente más larga que DOM a los lados de los tergos y sobre los esternos donde terminan generalmente curvados ( $>2$ DOM). 
Estructura. Cabeza (en vista frontal) tan larga como ancha. Gena un poco más ancha que el diámetro del ojo.

Tipos. Holotipo hembra PUERTO RICO, Utuado, Barrio Sabana Grande, sector Los Vázquez, carr[etera] 611, km 4, N18 16'39.30", W6641'52.00”, $238 \mathrm{~m}$ sweeping plants, 6.ii.2010, col. X. Labiosa (NMNH). Paratipos: PUERTO RICO, Maricao, Monte del Estado (State Forest), 425 rd, km 14.4, N18¹0'36.36”, W6657'42.87’, 21.iv.2010, $442 \mathrm{~m}$ asl, light trap and beating plants at night, col. N. Franz and Biol. 4446 (2, NMNH, JAG); Lajas, Laguna de Cartagena, NWR, N18 $8^{\circ} 00^{\prime} 40.28^{\prime}$ ', W67'06'10.23', 27.ii.2010, $10 \mathrm{~m}$ asl, col. Biol. 4446 and N. Franz (1, NMHN); Guánica, Bosque Estatal de Guánica at night (Hg light), N1758'20.06”, W66 52’0.69', 25.v.2010, 150 m asl, col. G. Goemans, A. Montoya and A. Vélez (1, UPRM).

Etimología. Nombre en alusión a la isla de origen, Borinquén que es el nombre nativo de la isla de Puerto Rico.

Macho. Desconocido.

Distribución. Isla de Puerto Rico.

\section{LISTA ANOTADA DE LAS ESPECIES ANTILLANAS DEL GÉNERO HYLAEUS.}

Hylaeus (Hylaeana) extrinsecus Snelling, 1982.

Distribución. Jamaica.

Hylaeus (Hylaeana) formosus Krombein, 1953.

= Hylaeus metopii Mitchell, 1960. North Carolina Agric. Exp. Sta. Tech. Bull. 141: 80.

=Hylaeus sp. A y C Alayo, 1976. Serie Biológica, Cuba 68: 18.

Distribución. Las Bahamas (isla Bimini, cayo Easter); cayos de La Florida (cayo Largo e isla Stock) y Cuba (registro nuevo).

Registro nuevo. CUBA: Tortuguilla, Guantánamo, v.1969, col. I. García (3 hembras, JAG); Cabo Corrientes, Guanahacabibes, Pinar del Río, v.1956, col. P. Alayo (3 hembras, JAG);

Cupeyal, Yateras, Guantánamo, vi. 64, vi.1966, col. P. Alayo (1 hembra, 1 macho, JAG).

Hylaeus (Hylaeana) phaeoscapus Snelling, 1982.

Distribución. Jamaica.

Hylaeus (Hylaeana) rawi Snelling, 1982.

Distribución. Jamaica. 
Hylaeus (Hylaeana) royesi Raw, 1984.

Distribución. Jamaica y Cuba (registro nuevo).

Registro nuevo. CUBA, cayo Coco, N Camagüey [actual Ciego de Ávila], 14-17, xii.96, col. M. López, Malaise trap 1 (hembra, JAG); cayo Coco [Ciego de Ávila], 11.vii.94, col. M. López (hembra, JAG); Yayal, Holguín, v.89, col. J. A. Genaro (hembra, JAG); Santa Clara, Las Villas [actual Villa Clara], iii.71 (macho, JAG); Jardín Botánico de Cienfuegos, vi.89 (macho, JAG).

Material examinado: JAMAICA, Try. Duncans, 23.viii.66, cols. Howdem y Becker (3 hembras y 1 macho, al cual se le extrajo y estudió la terminalia, CNC).

Nota. Un nido de esta especie (como Hylaeus sp.) fue encontrado en el Jardín Botánico de Cienfuegos, Cuba, en el interior de un palito de bambú. El parasitoide Cleonymus sp. (Hymenoptera: Pteromalidae) emergió de una celdilla (Genaro, 1996).

Hylaeus (Hylaeana) sp. 1.

Distribución. Islas Vírgenes Británicas (Isla Guana).

Nota. Snelling (1993) encontró esta especie nueva, la cual permanece sin describir (Snelling, 2005). Este constituye el primer registro nativo del género para el Gran Puerto Rico (Genaro y Franz, 2008).

Hylaeus (Hylaeana) sp. 2.

Distribución: Cuba. Una hembra recolectada en El Toldo, Moa, Nipe-Sagua Baracoa [Holguín] (JAG).

Hylaeus (Hylaeana) sp. 3.

Distribución. Hispaniola. Una hembra recolectada en Cabo Rojo, Pedernales, República Dominicana $(\mathrm{CNC})$.

Hylaeus (Prosopis) dearmasi sp. nov.

Distribución. Cuba y posiblemente Las Bahamas (Isla San Salvador). Según R. R. Snelling, com. pers., 1999) un macho recolectado en Las Bahamas (depositado en LACM) puede pertenecer a la misma especie.

Hylaeus (Prosopis) hispaniolensis sp. nov.

Distribución. Hispaniola.

Hylaeus (Prosopis) limbifrons (Cresson, 1869). 
$=$ Hylaeus sp. B Alayo, 1976. Serie Biológica, Cuba 68: 18.

Distribución. Cuba.

Hylaeus (Paraprosopis) wootoni (Cockerell, 1896)

Distribución. Suroeste de Estados Unidos y zonas adyacentes de México. Introducida en Islas Vírgenes Británicas (Isla Guana) (Snelling 1994, 2005; Genaro y Franz, 2008). La vía de introducción permanece desconocida.

\section{AGRADECIMIENTOS}

A Luis F. de Armas (Instituto de Ecología y Sistemática, La Habana) por la información brindada sobre las características del hábitat de $H$. dearmasi y las sugerencias brindadas durante la revisión crítica del manuscrito. Esteban Gutiérrez y Sandra Duarte ofrecieron información sobre los ejemplares de H. limbifrons depositados en el Museo Nacional de Historia Natural de Cuba. Cristina Juarrero amablemente dibujó los esternos de $H$. dearmasi y conformó las láminas. A Sam Droege (USGS Native Bee Inventory and Monitoring Program, Maryland), Nico Franz (actualmente en Arizona State University, EE. UU) y Hernán Torres (UPRM) por el préstamo de ejemplares. La colaboración mantenida con R. R. Snelling desde 1993 hasta su muerte en 2008 me permitió intercambiar información acerca de la historia natural de las abejas antillanas y sobre todo para este estudio, referente a las especies de Hylaeus.

\section{LITERATURA CITADA}

Alayo, P. 1973. Catálogo de los himenópteros de Cuba. Editorial Pueblo y Educación, Havana city. 218 pp.

Alayo, P. 1976. Introducción al estudio de los himénopteros de Cuba. IX- Superfamilia Apoidea. Serie Biológica, Instituto de Zoología, Cuba 68: 1-35.

Armas, L. F. de. 1976. Escorpiones del archipiélago cubano. V. Nuevas especies de Centruroides (Scorpionida: Buthidae). Poeyana, 146: 1-55.

Genaro, J. A. 1996. Nest parasites (Coleoptera, Diptera, Hymenoptera) of some wasps and bees (Vespidae, Sphecidae, Colletidae, Megachilidae, Anthophoridae) in Cuba. Caribbean Journal of Science, 32: 239-240.

Genaro, J. A. 2009. Especie nueva de Coelioxys (Hymenoptera: Apoidea: Megachilidae) para la Hispaniola. Novitates Caribaea, 2: 12-16.

Genaro, J. A. y N. Franz. 2008. The bees of Greater Puerto Rico (Hymenoptera: Apoidea: Anthophila). Insecta Mundi, 40: 1-24.

Harris, R. A. 1979. A glossary of surface sculpturing. Occasional Papers in Entomology, Department of Food and Agriculture, Division of Plant Industry, California, 28: 1-31.

Mitchell, T. B. 1960. Bees of the Eastern United States. Volume I. North Carolina Agricultural Experiment Station Technical Bulletin, 41: 1-538. 
Snelling, R. R. 1993. Collecting on Guana Island, British Virgin Islands and Puerto Rico. Melissa, 6: 5-6.

Snelling, R. R. 1994. Collecting on Guana Island. Melissa, 7: 15.

Snelling, R. R. 2005. Wasps, ants, and bees: Aculeate Hymenoptera. Pp. 283-296. In J. Lazell (Ed.) Island: Fact and Theory in Nature. University of California Press. 382 pp.

Urban, D. y J. S. Moure 2007. Hylaeini Viereck, 1916. Pp. 710 -723. In: J. S. Moure, D. Urban, y G. A. R. Melo (Orgs.). Catalogue of bees (Hymenoptera, Apoidea) in the Neotropical Region. Sociedade Brasileira de Entomologia, Curitiba. 1058 pp.

[Recibido: 14 de julio, 2016. Aceptado para publicación: 20 de agosto, 2016] 